\title{
From proteomic data to networks: statistics and methods reveal ciliary protein interaction landscape
}

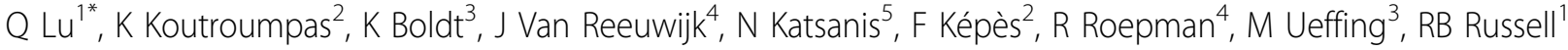 \\ From Cilia 2014 - Second International Conference \\ Paris, France. 18-21 November 2014
}

\section{Objective:}

The assembly of protein interaction networks (PIN) is an important step to understand the biological function of proteins. Affinity purification coupled to mass spectrometry (AP-MS) has become the technique of choice for the assembly and analysis of PINs. However, most current studies, especially in human cells, are focused on specific biological systems (e.g. the cilium) resulting in datasets of a small to intermediate scale. In such cases, methods that developed for genome-scale datasets are of limited utility. We propose here a framework that is specifically designed for the analysis of incomplete proteomic data focused on ciliary function and ciliopathies.

\section{Methods:}

The proposed framework consists of three steps. Initially, a revised Socio-Affinity algorithm [1] is applied to quantify the pairwise protein interaction affinities. After filtering hits from noise the constructed PIN is mined for protein clusters using a novel graph-clustering algorithm. Finally, Principle Component Analysis (PCA) is used to assess the quality of detected complexes.

\section{Results:}

We applied our algorithm to data from more than 400 TAP-MS experiments, using over 200 ciliary genes as baits. Weighted PINs consisting of low, medium and high confidence interactions were extracted from the data, and for each network a set of protein complexes is reported. Several known ciliary complexes have been successfully identified, while novel ciliary complexes are predicted.

\section{Conclusion:}

We demonstrate a computational framework that can deal with context specific proteomic data. Application to experimental data focusing on cilia provides a ciliary protein interaction landscape with the ciliary biological processes/functions in the centre.

Equal contribution by Q. Lu and K. Koutroumpas

\section{Authors' details \\ ${ }^{1}$ Cell Networks, Bioquant, University of Heidelberg, Heidelberg, Germany. ${ }^{2}$ Institute of Systems and Synthetic Biology, Genopole, CNRS, Université d'Evry, Evry, France. ${ }^{3}$ Division of Experimental Ophthalmology and Medical Proteome Center, Eberhard-Karls Universität Tübingen, Tübingen, Germany. ${ }^{4}$ Department of Human Genetics and Radboud Institute for Molecular Life Sciences, Radboud University Medical Center, Nijmegen, The Netherlands. ${ }^{5}$ Center for Human Disease Modeling, Department of Cell Biology, Duke University, Durham, NC, USA.}

Published: 13 July 2015

Reference

1. Gavin AC, Aloy P, Grandi P, Krause R, Boesche M, Marzioch M, et al: Proteome survey reveals modularity of the yeast cell machinery. Nature 2006, 440(7084):631-636.

doi:10.1186/2046-2530-4-S1-P90

Cite this article as: Lu et al:: From proteomic data to networks: statistics and methods reveal ciliary protein interaction landscape. Cilia 20154 (Suppl 1):P90. 